\title{
Unilateral Unique Primary Melanomas
}

\author{
Tchernev $\mathbf{G}^{1,2 *}$, Chokoeva $\mathrm{AA}^{3}$ \\ ${ }^{1}$ Onkoderma"-Policlinic for Dermatology and Dermatologic Surgery, Sofia, Bulgaria \\ ${ }^{2}$ University Hospital Lozenetz, Policlinic for Dermatology and Venereology, Sofia, Bulgaria \\ 3"Onkoderma"- Policlinic for Dermatology and Dermatologic Surgery, Sofia, Bulgaria
}

Received: December 19, 2015; Accepted: December 24, 2015; Published: December 27, 2015

*Corresponding author: Associate Professor Georgi Tchernev, PhD, Policlinic for Dermatology and Venereology, University Hospital Lozenetz, Koziak street 1, 1407 Sofia, Bulgaria, Tel: +359 885588 424; E-mail: georgi_tchernev@yahoo.de

\section{Description}

We present a 68-year-old female patient, consulted on the occasion of a pigment lesion at her left cheek with 7-8 years old history. Clinical and dermatoscopically, the lesion corresponded perfectly to the criteria for nodular malignant melanoma. The performed imaging and serological diagnostics showed no evidence of metastases. Within the clinical examination, our attention was further drawn to the availability of additional pigment lesion localized at the heel area - measuring $1 \mathrm{~cm}$ to 0.8 $\mathrm{cm}$, symmetrical, with uniform color, clearly distinguished from the healthy tissue, without evidence of elevation, which was strongly suspected for superficial spreading melanoma (Figure 1a-c). A surgical eradication was planned with field of surgical security of $1 \mathrm{~cm}$ in all directions.

Unilateral and multiple manifestation of heterogeneous type melanomas (nodular, superficial spreading melanoma, amelanotic melanoma, desmoplastic melanoma etc.) occurs more frequent in patients with FAMMM syndrome (familial or sporadic form) or hereditary melanoma [1, 2].

About $10 \%$ of all melanoma patients report a family history of melanoma; however, individuals with features of true hereditary melanoma (ie, unilateral lineage, multigenerational, multiple primary lesions, and early onset of disease) are in fact quite rare [1]. Although many new loci have been implicated in hereditary melanoma, CDKN2A mutations remain the most common [1]. These patients have a high risk of developing multiple

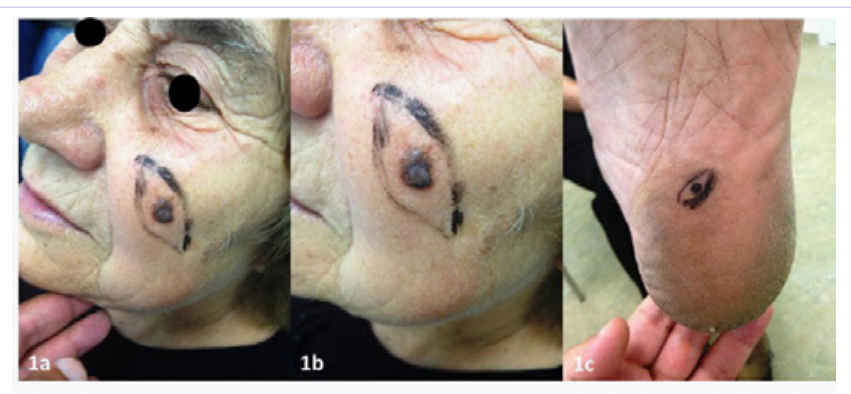

Figure 1: a,b: Clinical manifestation of the nodular pigmented tumor with uniform color, clearly distinguished from the healthy tissue, hightly suspected for nodular malignant melanoma, located on the left cheek, bellow os zygomaticus in 68-year-old female patient.

1c: Clinical manifestation of a superficial spreading melanoma, located on the heel area of the left leg in the same patient. primary melanomas and internal organ malignancies, especially pancreatic cancer; therefore, a multidisciplinary approach is necessary in many cases [1]. The value of dermoscopic examination and total body photography performed at regular intervals has been suggested by a number of studies, and should therefore be considered for these patients and their first-degree relatives [1,2].

The simultaneous, and furthermore, the unilateral location of heterogeneous types of melanomas in adult patients have been sporadically described in the literature, but it remains rare in general [3].

Regarding the newest investigations about the MPM (multiple primary melanomas) the risk of a subsequent melanoma decreased from $2 \%$ in the first year after diagnosis to a stable approximately $1 \%$ rate through 15 years of follow-up [4]. The risk of MPMs, although highest in the first year after diagnosis, remains stable thereafter [4].Those at highest risk of MPMs are older, male, white, and partnered [4]. Clinicians should be aware of the rate of MPMs and recognize high-risk subgroups [4].

\section{References}

1. Soura E, Eliades PJ, Shannon K, Stratigos AJ, Tsao H. Hereditary melanoma: Update on syndromes and management: Genetics of familial atypical multiple mole melanoma syndrome. J Am Acad Dermatol. 2016;74(3):395-407. doi: 10.1016/j.jaad.2015.08.038.

2. Tchernev G, Ananiev J, Cardoso JC, Chokoeva AA, Philipov S, Penev $\mathrm{PK}$, et al. Multiple primary cutaneous melanomas in patients with FAMMM syndrome and sporadic atypical mole syndrome (AMS): what's worse? Wien Med Wochenschr. 2014;164(15-16):302-307. doi: 10.1007/s10354-014-0295-8.

3. Krajewski AC, Hart DR, Hieken TJ. Multiple primary melanoma in the elderly. Am J Surg. 2016;211(1):84-88. doi: 10.1016/j. amjsurg.2015.05.023.

4. Moore MM, Geller AC, Warton EM, Schwalbe J, Asgari MM. Multiple primary melanomas among 16,570 patients with melanoma diagnosed at Kaiser Permanente Northern California, 1996 to 2011. J Am Acad Dermatol. 2015;73(4):630-636. doi: 10.1016/j.jaad.2015.06.059

Citation: Tchernev G, Chokoeva AA (2015) Unilateral Unique Primary Melanomas. Clin Res Dermatol Open Access 2(3): 1-1. 